\title{
Micellization of quaternized poly(2-(dimethylamino)ethyl methacrylate)- block-poly(methyl methacrylate) copolymers in water
}

\author{
S. Antoun, J. -F. Gohy and R. Jérôme \\ Center for Education and Research on Macromolecules (CERM) - Institute of Chemistry B6, University of \\ Liège, Sart-Tilman, B-4000 Liège, Belgium
}

\begin{abstract}
Micellization of a series of amphiphilic quaternized poly(2-(dimethylamino)ethyl methacrylate)block-poly (methyl methacrylate) (PQDMAEMA-b-PMMA) copolymers has been studied in water. The alkyl halide used for the quaternization of the aminated block has an effect on the solution properties of the diblocks, that have been investigated by dynamic light scattering and surface tension measurements. When a short length alkyl halide is used, the diblock copolymers behave like traditional amphiphiles, whereas their behavior is similar to polysoaps in case of long alkyl halides. The size of the micelles increases when 1-bromopropan-3-ol is substituted for 1-bromopropane in the quaternization reaction. It is, however, independent of the structure of butyl bromide, i.e. $n$-butyl vs tert-butyl bromide. The solution properties of a star-shaped PQDMAEMA- $b$ PMMA copolymer have also been studied and compared to the linear counterpart. The star-shaped architecture actually prevents the block copolymer to be properly oriented at the air/water interface in agreement with the formation of unimer micelles.
\end{abstract}

Keywords: Block copolymer; Star copolymer; Cationic polymeric surfactant

\section{Introduction}

The self-association of amphiphilic block copolymers in a selective solvent for one constituent is well documented in the scientific literature [1-4]. Micelles are formed, that consists of a core formed by the insoluble blocks surrounded by a shell of solvated blocks. Much attention is paid to the micellization of amphiphilic copolymers in water because these systems have potential in several application fields, such as paints and coatings (electrosteric stabilization of latex particles [5] and pigments [6]) and galenics (micelles serving as drug carriers) $[7-8]$.

Representative examples of amphiphilic copolymers studied for their micellization ability in aqueous media are block copolymers of the following comonomer pairs: ethylene oxide/butylene oxide [9-11], ethylene oxide/propylene oxide [12-20], ethylene oxide/styrene [21-22], quaternized 4-vinylpyridine/styrene [23-28] and methacrylic acid/styrene [29-35]. A review of this topic was published by Selb and Gallot in 1985 [36]. More recently, micelles with remarkable morphologies including rods, lamellae, vesicles, and tubules were observed, being entropically driven whenever the chains of the core-forming blocks are less stretched than in dense spherical aggregates [37]. Eisenberg et al. reported on the formation of the so-called 'crew-cut' aggregates, formed by highly asymmetric diblock copolymers consisting of a short hydrophilic polyelectrolyte or neutral block and a long hydrophobic polystyrene or polybutadiene block [38-49]. These aggregates were actually formed by the addition of a small amount of water to the copolymer dissolved in a non-selective solvent, e.g. dimethylformamide. They were kinetically frozen by the removal of the non-selective organic solvent by dialysis against the selective one, i.e. water. In contrast to the amphiphilic diblocks used by Eisenberg et al., the major block of the copolymers studied in this work is the hydrophilic block, which results in the complete solubility of the polymeric amphiphiles in water. A series of poly (2-dimethylamino)-b-poly(methyl methacrylate)

(PQDMAEMA- $b$-PMMA) diblocks will be synthesized by sequential living anionic polymerization of the comonomers. For the sake of comparison, a star-shaped counterpart of one diblock will also be made available. Upon quaternization of the polyaminated block, all these copolymers will be soluble in water and their micellar solution properties will be studied in relation to the quaternizing agent used (length and branching of the alkyl chain) and the molecular architecture of the diblocks (linear and star-shaped).

Micellization of this type of PDMAEMA- $b$-PMMA diblock was studied by Baines et al. [50]. Critical micelle concentration was found to increase with the length of the hydrophobic block, the length of the hydrophilic block being constant, whereas the size and the aggregation numbers of the micelles decreased. When the molar mass of the copolymer was increased at constant composition, the $\mathrm{cmc}$ and the micelle size increased in parallel, in contrast to the association number which decreased. The complete protonation of the DMAEMA component by 
$\mathrm{HCl}$ prevented micellization from occurring, except in the presence of salt which screened the charge repulsion of the polycationic blocks.

In this study, attention will be paid to the quaternization of the PDMAEMA block by different alkyl halides. In case of long alkyl chains, formation of a polysoap-like system is expected [51-55], as result of intramolecular hydrophobic interaction of the pendant alkyl chains leading to hydrophobic microdomains. So, two distinct levels of organization might be observed upon micellization, i.e. formation of a hydrophobic PMMA core surrounded by a corona of quaternized polyaminated blocks, in which a subaggregation of the hydrophobic alkyl chains should occur. This type of system has potential in the design of functional nanomaterials, as recently shown by Ikkala et al. who studied bulk poly(4-vinylpyridine)-block-polystyrene diblocks, modified by the complexation of the 4-vinylpyridine units by a low molar mass surfactant [56].

\section{Experimental section}

\subsection{Copolymer synthesis}

The diblock copolymers were anionically synthesized as reported elsewhere [57]. Briefly, the monomers were purified by distillation over triethylaluminum. The glass reactor containing the required amount of $\mathrm{LiCl}(10 / 1$ $\mathrm{LiCl} /$ initiator molar ratio) was flame dried under vacuum, purged with nitrogen, added with the solvent (tetrahydrofuran, THF) and cooled down to $-78^{\circ} \mathrm{C}$. Diphenylmethyllithium was added dropwise until a persistent orange color was observed, followed by the required amount of this initiator. Methylmethacrylate was first polymerized at $-78^{\circ} \mathrm{C}$ for $1 \mathrm{~h}$, followed by DMAEMA $\left(2 \mathrm{~h}\right.$ at $\left.-78^{\circ} \mathrm{C}\right)$. A sample was picked out from the reactor before the addition of DMAEMA. The copolymerization reaction was then quenched with methanol.

A star-shaped copolymer was synthesized by the method reported by Rempp et al. [58]. In this case, DMAEMA was first polymerized, followed by MMA. An aliquot of the living diblock copolymer was then picked out, deactivated by methanol and further used as the copolymer 'arm'. A difunctional monomer (i.e. ethyleneglycol bis-methacrylate) was then added to the living diblock macroanions with formation of the star-shaped copolymer [58].

The final product was characterized by size exclusion chromatography (SEC) and nuclear magnetic resonance (NMR). SEC was performed in tetrahydrofuran, with a Hewlett-Packard 1050 liquid chromatograph equipped with two Plgel columns (1000 and $10000 \AA$, respectively) and a Hewlett-Packard 1047A refractive index detector. The Hewlett-Packard 79994A software was used for data analysis. Poly(methylmethacrylate) standards were used for calibration. A $1 \mathrm{wt} \%$ solution of the polymer in THF was filtered before injection. ${ }^{1} \mathrm{H}$ NMR spectra of $1 \mathrm{wt} \%$ solution in fully deuterated THF were recorded at $400 \mathrm{MHz}$ with a Bruker AM 400 spectrometer. THF is known to be a non-selective solvent for the diblocks [50, 57]. This was verified by dynamic light scattering (DLS), which confirmed the absence of aggregates, and by ${ }^{1} \mathrm{H}$ NMR, which showed well-resolved signals for the two blocks. $\overline{\boldsymbol{M}}_{\text {Lof }}$ the second block was calculated from the ${ }^{1} \mathrm{H}$ NMR spectrum of the copolymer and $\overline{\boldsymbol{M}}_{\text {Lnof }}$ the first block. The apparent mean number of arms $\left(n_{\mathrm{r} w} 4\right)$ in the star-shaped copolymer was calculated from the molecular weights for the constitutive arm and the copolymer, respectively. These molecular weights were determined by SEC with PMMA standards. The molecular characteristic features are listed in Table 1.

Table 1. Molecular characteristic features of the samples used in this study

\begin{tabular}{|c|c|c|c|c|c|c|}
\hline \multirow[t]{2}{*}{ Copolymer composition (NMR) wt' } & \multicolumn{2}{|c|}{ First block (SEC) } & \multicolumn{2}{|c|}{ Copolyner (NMR) } & \multirow[t]{2}{*}{ Quatenization by alkyl bromide } & \multirow[t]{2}{*}{ Sample } \\
\hline & $M_{w} \times 10^{-5}$ & $M_{W} / M_{I}$ & $M_{\mathrm{w}} \times 10^{-3}$ & $M_{W} / M_{2}$ & & \\
\hline \multirow[t]{4}{*}{ PMMA-b-PDMAEMA $15 / 85$} & 1.0 & 1.2 & 6.6 & 1.15 & unquafernized & $Q_{0}$ \\
\hline & 1.0 & 1.2 & 6.6 & 1.15 & ehyl & $Q_{2}$ \\
\hline & 1.0 & 1.2 & 6.6 & 1.15 & pentyl & $Q_{5}$ \\
\hline & 1,0 & 1.2 & 6.6 & 1.15 & heptyl & $Q_{T}$ \\
\hline \multirow[t]{5}{*}{ PMMA-b-PDMAEMA $16 / 84$} & 3.85 & 1.05 & 34.0 & 1.1 & n-propyl & $Q_{3}$ \\
\hline & 3.85 & 1.05 & 24.0 & 1.1 & n-propyl-3ol & $Q_{301}$ \\
\hline & 3.85 & 1.05 & 24.0 & 1.1 & $n$ propyl-2.3 diol & Q3jiol \\
\hline & 3.85 & 1.05 & 240 & 1.1 & $n$ butyl & \\
\hline & 3.85 & 1.05 & 24.0 & 1.1 & tert-butyl & \\
\hline PDMAFMA- $b$ PMMA7R/2? & 4.4 & 1.05 & 5.65 & 1.1 & ethyl & ${ }^{*} \mathrm{AQ2}$ \\
\hline (1m) 7802 & - & - & 21.10 & 1.45 & ethyl & ${ }^{*} \mathrm{SQ}_{2}$ (dax) \\
\hline
\end{tabular}


The PDMAEMA block of the copolymers was quaternized by several alkyl bromides (RBr). In a typical experiment, the PMMA- $b$-PDMAEMA diblock was dissolved in tetrahydrofuran and added with an excess of quaternizing agent. The solution was heated at $40^{\circ} \mathrm{C}$ for $24 \mathrm{~h}$, and the quaternized product was collected after the solvent removal. Known amounts of PQDMAEMA- $b$-PMMA copolymers were dissolved in water and purified by dialysis against regularly replaced distilled water. The molecular characteristics of all the quaternized copolymers analyzed in this study are listed in Table 1. The degree of quaternization was determined by titrating the non-quaternized amine groups with standard $\mathrm{HCl}$ solution and the quaternization reaction was found to be quantitative in all the cases. The accuracy of the method was checked with ${ }^{1} \mathrm{H}$ NMR as described elsewhere [59].

\subsection{Solution properties}

All the solutions were prepared by dilution of the dialyzed copolymer solutions by bidistilled water containing $\mathrm{NaCl}(0.1 \mathrm{~mol} / \mathrm{l})$.

Surface tension $(\gamma)$ was measured with a thermostated Kruss tensiometer equipped with a calibrated platinum plate. All measurements were carried out at $20^{\circ} \mathrm{C}$ and repeated several times for each solution. The surface tension of bidistilled water was regularly checked $(72-73 \mathrm{mN} / \mathrm{m})$.

Dynamic light scattering (DLS) was measured with a Brookhaven Instrument Corp. DLS apparatus that consisted of a BI-200 goniometer, a BI-2030 digital correlator and an Ar-ion laser (LEXEL Lasers) with a wavelength of $488 \mathrm{~nm}$. The scattering angle used for the measurements was $90^{\circ}$. A refractive index matching bath of filtered decalin surrounded the scattering cell, and the temperature was controlled at $25^{\circ} \mathrm{C}$. Prior to sample loading, appropriate glass vessels were soaked overnight in sulfochromic solution, thoroughly cleaned by bidistilled water and dried in a vacuum oven.

The experimental correlation function was measured, and the hydrodynamic diameter of the particles was calculated by using the Stokes-Einstein approximation [60]. When aggregates of different size are formed in solution, the experimental correlation function depends on all the individual decay processes. In this case, the data analysis was performed by using the coNTn routine which gives access to the distribution of the relaxation times in the experimental time correlation functions [60]. Z-averaged distribution of hydrodynamic diameter was then calculated.

\section{Results and discussion}

The surface tension of the aqueous solutions of the copolymers was measured with the purpose to determine the critical micelle concentration $(\mathrm{cmc})$. The average size of the micelles was measured by DLS. All the copolymers were readily soluble in water after purification by dialysis and no organic solvent was used further to solubilize them. This preparation method of the copolymer solutions is basically different from that one used in previous works. Indeed, Selb and Gallot [24] observed micellization of quaternized poly(4-vinylpyridine)-blockpolystyrene in methanol/water/salt mixtures the copolymers being first dissolved in methanol and then added with the aqueous electrolyte solution. When the water content of the solution was increased, the average degree of the copolymer association passed through a maximum. Similarly, Munk et al. investigated poly(methacrylic acid)-block-polystyrene copolymers, which formed micelles with polystyrene core in water [29-35]. These copolymers could not be directly dissolved in water but first in dioxane/water mixtures rich in dioxane. The micelles formed in this solvent mixture were transferred in pure water or in aqueous buffers by stepwise dialysis. Tuzar et al. showed that the size of these micelles increased with the dioxane content of the mixed solvent, apparently because of the swelling of the polystyrene core [35]. Baines et al. also used a non-selective organic solvent in a preliminary dissolution step of PDMAEMA- $b$-PMMA copolymers, thus prior to micellization in water [50]. Formation of large aggregates was observed when these copolymers were directly dissolved in water or in mixed aqueous solutions, in contrast to what happened when the 'two-step' dissolution in water was used.

In this study, copolymers containing a major hydrophilic block $(>70 \mathrm{wt} \%)$ can be directly dissolved in water, with formation of optically clear micellar solutions, which does not mean that the micelles are close to the thermodynamic equilibrium, particularly at temperature below the glass transition temperature of the coreforming blocks. Nevertheless, residual THF could contaminate the PMMA core and plasticize it after the quaternization reaction. However, THF is removed during the dialysis step, and the micelles are finally 'frozen' in pure water. 


\subsection{Effect of the alkyl bromide used as the quaternizing agent on the cmc}

Fig. 1 shows how the surface tension of aqueous solutions of the PMMA- $b$-DMAEMA 15/85 diblock (Table 1) depends on concentration before $\left(\mathrm{Q}_{0}\right)$ and after quaternization of the PDMAEMA block by ethyl bromide $\left(\mathrm{Q}_{2}\right)$, pentyl bromide $\left(\mathrm{Q}_{5}\right)$ and heptyl bromide $\left(\mathrm{Q}_{7}\right)$, respectively. The $\mathrm{Q}_{0}$ and $\mathrm{Q}_{2}$ samples show the typical behavior of surfactants, which allows the cmc to be determined unambiguously (Table 2). The copolymer concentration needed to observe the micelle formation is, however, one order of magnitude higher for the ionized $\mathrm{Q}_{2}$ sample, whereas the surface tension at the $\mathrm{cmc}$ is higher for $\mathrm{Q}_{2}$ (ca $55 \mathrm{mN} / \mathrm{m}$ ) than for $\mathrm{Q}_{0}(\mathrm{ca} 40 \mathrm{mN} / \mathrm{m})$. This observation suggests that the chain conformation at the air/water interface differs considerably as result of quaternization of the PDMAEMA block. The higher surface tension observed for $\mathrm{Q}_{2}$ at and above the $\mathrm{cmc}$ is in agreement with the electrostatic repulsion between the charged PDMAEMA blocks, so leading to a low packing density at the air/water interface and to a small decrease in the water surface tension. In this respect, Baines et al. did not observe the micellization of PDMAEMA- $b$-PMMA diblock quaternized by $\mathrm{HCl}$, because of the electrostatic repulsion of the protonated PDMAEMA blocks [61]. This effect is not operative in the $\mathrm{Q}_{0}$ solution and the diblock chains are better organized at the water surface, more likely with the PDMAEMA blocks dangling in the water phase and the PMMA blocks pointing out to the air. Therefore, a lower surface tension is observed at the cmc $(40 \mathrm{mN} / \mathrm{m})$ which is smaller $(0.3 \mathrm{~g} / \mathrm{l})$ compared to the $\mathrm{Q}_{2}$ sample. These observations are in complete agreement with the well-known decrease in the cmc whenever the charge density of the hydrophilic component is decreased [62].

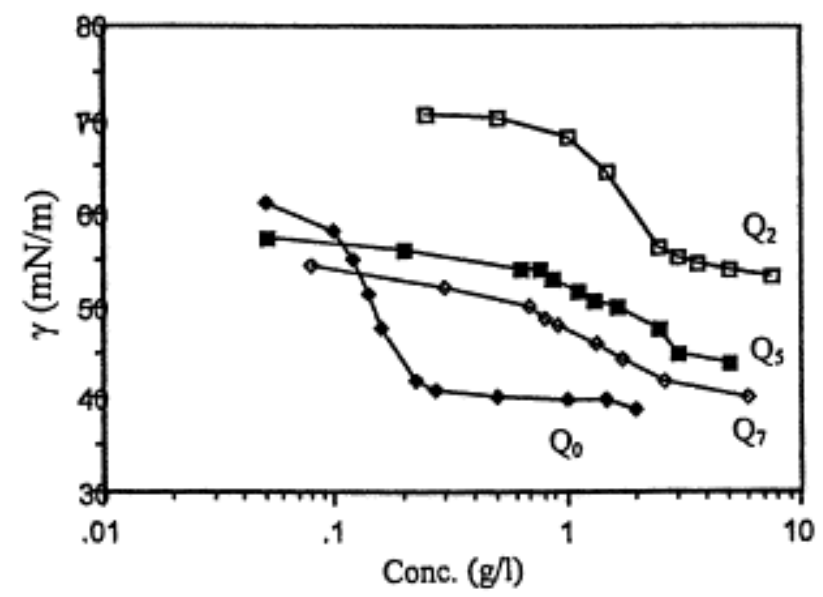

Fig. 1. Concentration dependence of the surface tension of aqueous solutions of the PMMA- $b$-PDMAEMA $15 / 85$ diblock (Table 1 ) non-quaternized $\left(\mathrm{Q}_{0}\right)$ and quaternized by different alkyl bromides $\left(\mathrm{Q}_{2}, \mathrm{Q}_{5}\right.$ and $\left.\mathrm{Q}_{7}\right)$. All the solutions are added with $\mathrm{NaCl}(0.1 \mathrm{M})$

Table 2. Variation of the hydrodynamic diameter of the aggregates (micelles) with copolymer concentration, in the presence of $\mathrm{NaCl}(0.1 \mathrm{M})$ for the $Q_{0}, Q_{2}, Q_{5}$ and $Q_{7}$. The corresponding cmc and $\gamma_{\mathrm{cmc}}$ are mentioned (* too low intensity for being measured accurately)

\begin{tabular}{lcccc}
\hline Cone, (g/) & \multicolumn{4}{c}{ Hydrodyramic dimeter (m) } \\
\cline { 2 - 5 } & $\mathrm{Q}_{0}$ & $\mathrm{Q}_{2}$ & $\mathrm{Q}_{5}$ & $\mathrm{Q}_{7}$ \\
\hline 0,05 & 12 & $*$ & 352 & 398 \\
0.10 & 19 & $*$ & 365 & 410 \\
0.25 & 19 & $*$ & 350 & 425 \\
0.50 & 34 & 46 & 360 & 450 \\
1.50 & 23 & 61 & 333 & 435 \\
3.0 & 36 & 59 & 365 & 422 \\
$\operatorname{cmc}(\mathrm{g} / \mathrm{l})$ & 0.3 & 2.2 & - & - \\
$\gamma_{\text {ran }}(\mathrm{mN} / \mathrm{m})$ & 40 & 55 & - & - \\
\end{tabular}


A well-defined cmc and the independence of the surface tension on the equilibration time are consistent with the formation of micelles at equilibrium in the case of low molecular weight diblock containing a very short hydrophobic block $\left(\bar{M}_{\mathrm{n}}=1000\right)$.

When the bromide used for the quaternization of the PDMAEMA block contains longer alkyl radicals $\left(\mathrm{Q}_{5}\right.$ and $\mathrm{Q}_{7}$ samples), the surface activity of the diblock is much less pronounced (Fig. 1), the behavior being quite reminiscent of polysoaps. The pentyl and heptyl chains in $\mathrm{Q}_{5}$ and $\mathrm{Q}_{7}$, respectively, are expected to form small hydrophobic micro-domains along the polyelectrolyte PQDMAEMA chains. This intramolecular substructure is similar to that one observed in polysoaps or in the so-called 'surf-complexes' formed by low molecular weight surfactants in interaction with mutually reactive water-soluble polymers [63-64]. These 'complexes' generally adopt an hypercoiled conformation, which may explain that the $\mathrm{Q}_{5}$ and $\mathrm{Q}_{7}$ unimers are not properly oriented at the air/water interface and that no clear cmc is observed. Therefore, the $\mathrm{Q}_{5}$ and $\mathrm{Q}_{7}$ chains have to be regarded rather as 'polymeric aggregates' than as unimers. However, these 'polymeric aggregates' that are formed at low concentration $(0.05 \mathrm{~g} / \mathrm{l})$ can migrate to the air/water interface with the hydrophobic components pointing out to the air phase, thus reducing to some extent the surface tension of the solution, but in a way that does not depend very much on the copolymer concentration.

The hydrophobic PMMA blocks tend also to self-assemble intermolecularly into hydrophobic micro-domains, which results in additional complexity in the structure of the micellar aggregates formed by the $\mathrm{Q}_{5}$ and $\mathrm{Q}_{7}$ samples.

When the PDMAEMA- $b$-PMMA (16/84) diblock (Table 1 ) is quaternized by $n$-propyl bromide, 1-bromopropan3-ol, 1-bromopropan-2,3-diol, n-butyl bromide and tert-butyl bromide $\left(\mathrm{Q}_{3}, \mathrm{Q}_{3 \mathrm{ol}}, \mathrm{Q}_{3 \text { diol }}, \mathrm{Q}_{\mathrm{n}}\right.$ and $\mathrm{Q}_{\mathrm{t}}$ samples $)$ no sharp decrease in the surface tension is noted, as was the case for the $\mathrm{Q}_{5}$ and $\mathrm{Q}_{7}$ samples. However, the relatively short length of the pendant hydrophobic segments could hardly explain the formation of hydrophobic microdomains along the polymer chains, as was stated for the $\mathrm{Q}_{5}$ and $\mathrm{Q}_{7}$ samples. Therefore, the lack of clear-cut cmc in these samples of higher molecular weight $\left(\overline{\boldsymbol{M}}_{\mathrm{L} \text { of }} 24,000\right)$ compared to the first series $\left(\overline{\boldsymbol{M}}_{\mathrm{L}}\right.$ of 7600$)$ is not evidence that they behave as polysoaps, because kinetic restrictions related to decreased chain mobility and dynamics might control the migration to the surface and the orientation at the air/water interface. Actually, it is well-known in the scientific literature that short polymer chains migrate much faster than longer ones to the interface [65]. Moreover, the equilibration time before any measurement has a strong effect as illustrated in the next section. So, the apparent absence of a clear-cut cmc might result from an exceedingly long time to reach the equilibrium conformation at the interface.

\subsection{Effect of the alkyl bromide used as the quaternizing agent on the hydrodynamic diameter measured by DLS}

Table 2 lists the hydrodynamic diameter measured by DLS for the $\mathrm{Q}_{0}, \mathrm{Q}_{2}, \mathrm{Q}_{5}$ and $\mathrm{Q}_{7}$ samples, at different copolymer concentrations. These values have been calculated on the basis of the Stokes-Einstein approximation and extrapolated to zero concentration. The $\mathrm{Q}_{0}$ sample forms micelles with a diameter of ca $10 \mathrm{~nm}$, which is in complete agreement with the value reported by Baines et al. for micelles formed by comparable PDMAEMA- $b$ PMMA diblocks in $\mathrm{MeOH} /$ water and THF/water mixtures [50]. Baines et al. studied the influence of the solution preparation on the final hydrodynamic diameter measured by DLS. The diblock copolymers were either directly dissolved in water or dissolved in a non-selective cosolvent (THF or methanol) followed by dilution with water. The micelle diameters were found to be independent of the preparation method only for low molar mass copolymers containing less than 30 mol\% PMMA. These criteria are actually fulfilled for the $\mathrm{Q}_{0}$ sample, which strengthens the opinion that the behavior of the $\mathrm{Q}_{0}$ sample is typical of traditional surfactants.

As mentioned in the experimental part, the micellization of the block copolymers was studied in water added with $\mathrm{NaCl}(0.1 \mathrm{M})$. Indeed, Baines et al. observed that no micelle was formed by the fully protonated PDMAEMA- $b$-PMMA copolymers directly dissolved in water $[50,61]$ However, micellization occurred upon the addition of salt that screened the electrostatic repulsion of the cationic charges on the PDMAEMA chains. Similar observations were reported by Selb and Gallot for quaternized poly(4-vinylpyridine)-block-polystyrene [26] and by Astafieva et al. for poly(styrene)-block-poly( $\mathrm{Na}$ acrylate) [66], bigger micelles being formed as the salt concentration was increased. $0.01 \mathrm{M}$ aqueous $\mathrm{KCl}$ solution was used by Baines et al. to observe micellization of the PDMAEMA- $b$-PMMA copolymers at a $\mathrm{pH}$ of 2 . At $\mathrm{pH}$ as high as 9.5, addition of salt was still required to promote micellization, although the degree of protonation of the PDMAEMA block must be very low under these conditions. In this work, all the measurements were carried out in $0.1 \mathrm{M} \mathrm{NaCl}$ solution in water. 
Micelles with a diameter of ca $40 \mathrm{~nm}$ are formed by the $\mathrm{Q}_{2}$ sample at concentration exceeding the $\mathrm{cmc}$. These micelles are much larger than those ones formed by the unquaternized copolymer $\left(\mathrm{Q}_{0}\right)$ under the same conditions. Thus, an increase in the charge density of the water-soluble block leads to bigger micelles, all the other parameters being kept constant. This observation is in agreement with the data published by Zhang et al. for the micelles formed by polystyrene-block-poly(acrylic acid) copolymers [67]. These authors observed that the stretching of the poly(acrylic acid) chains increased with the degree of neutralization and was extremely high when the neutralization was close to completion. This chain extension was, however, decreased by the addition of salt (Debye screening). Interestingly enough, Zhang et al. showed that the ionized chains in the corona of the micelles were more extensively stretched in the presence of salt than the same chains with a low degree of ionization. This stretching effect was theoretically predicted by Pincus, who suggested that the addition of electrolyte decreases the counterion osmotic pressure, which stretches the polymer chains [68].

The $\mathrm{Q}_{5}$ and $\mathrm{Q}_{7}$ samples form large size aggregates, which cannot be regarded as classical micelles and whose the size remains nearly constant in the whole concentration range.

No cmc is clearly detected for the $\mathrm{Q}_{3}, \mathrm{Q}_{3 \mathrm{ol}}$ and $\mathrm{Q}_{3 \text { diol }}$ samples, although DLS shows that micelles are formed (Table 3). This observation suggests that the lack of well-defined cmc might result from the higher molecular weight of the PDMAEMA- $b$-PMMA precursor, as discussed before. These micelles have larger diameters than the $\mathrm{Q}_{2}$ sample (Table 2 and Table 3). The micelle diameter increases when the propyl radical is modified by one hydroxyl group (from $\mathrm{Q}_{3}$ to $\mathrm{Q}_{3 \mathrm{ol}}$ ) and still further when two hydroxyl groups are attached to the propyl (from $\mathrm{Q}_{3 \mathrm{ol}}$ to $\left.\mathrm{Q}_{\text {3diol }}\right)$. Although no experimental evidence is available, this effect is thought to result from an increase in the aggregation number of the micelles rather than from the stretching of the corona. In this respect, hydrogenbonding of the hydroxyl groups could play a role in the aggregation mechanism.

The effect of the architecture of the quaternizing alkyl chain has also been examined in the $\mathrm{Q}_{\mathrm{n}}$ and $\mathrm{Q}_{\mathrm{t}}$ samples (Table 3). No clear dependence of the micelle diameter on the structure of the butyl chain is, however, observed.

Table 3. Dependence of the hydrodynamic diameter of the micelles on the copolymer concentration, for the $\mathrm{Q}_{3}$, $\mathrm{Q}_{3 \mathrm{ol}}, \mathrm{Q}_{3 \text { diol }}, \mathrm{Q}_{\mathrm{n}}$ and $\mathrm{Q}_{\mathrm{t}}$ samples, in the presence of $\mathrm{NaCl}(0.1 \mathrm{M})$

\begin{tabular}{lccccc}
\hline Conc. (a/) & \multicolumn{5}{c}{ Hydrodynamic diameter $(\mathrm{mm})$} \\
\cline { 2 - 6 } & $\mathrm{Q}_{3}$ & $\mathrm{Q}_{\text {3al }}$ & $\mathrm{Q}_{3 \text { do }}$ & $\mathrm{Q}_{1}$ & $\mathrm{Q}_{1}$ \\
\hline 0.05 & 55 & 66 & 98 & 73 & 69 \\
0.1 & 58 & 59 & 89 & 80 & 77 \\
0.5 & 54 & 72 & 93 & 75 & 53 \\
1.5 & 63 & 67 & 108 & 84 & 66 \\
3 & 58 & 74 & 104 & 79 & 78 \\
\hline
\end{tabular}

\subsection{Influence of the copolymer architecture on the aggregation behavior}

The $\mathrm{SQ}_{2}$ is not a true star because the extremities of the PMMA blocks are linked one to each other through 'microdomains' of crosslinked poly(ethylene glycol bis-methacrylate). This molecular architecture is supposed to reduce the chain mobility and, accordingly, to modify the solution properties of $\mathrm{SQ}_{2}$ compared to the arm precursor molecules (sample $\mathrm{AQ}_{2}$, in Table 1). The surface activity of star-shaped copolymers has rarely been studied [69,70]. Investigations are difficult because extensive equilibration times may be required [71], because of slow diffusion or conformational changes at the air/water interface.

In this study, surface tension was measured for the star-shaped $\mathrm{SQ}_{2}$ and the arm $\mathrm{AQ}_{2}$ at two equilibration times (15 min and $15 \mathrm{~h})$.

Fig. 2a shows the dependence of the surface tension on the copolymer concentration. The dependence observed for the arm copolymer $\mathrm{AQ}_{2}$ is comparable to the curve observed for classical surface-active compounds. The sharp break in the curve at a concentration of ca. $0.16 \mathrm{~g} / \mathrm{l}$ is assigned to the $\mathrm{cmc}$. The surface tension at the $\mathrm{cmc}$ is $50 \mathrm{mN} / \mathrm{m}$. Compared to $\mathrm{AQ}_{2}, \mathrm{SQ}_{2}$ shows a lower surface activity, indicating that the PMMA blocks have a restricted tendency to localize at the air/water interface. The surface tension decreases in such a way that no distinct break indicative of $\mathrm{cmc}$ is observed. Therefore, $\mathrm{SQ}_{2}$ behaves as a prefabricated micelle, that consists of a PMMA core surrounded by a corona of PQDMAEMA blocks. The low surface activity is in line with this picture, since micelles are known not being surface-active [72 and 73]. 

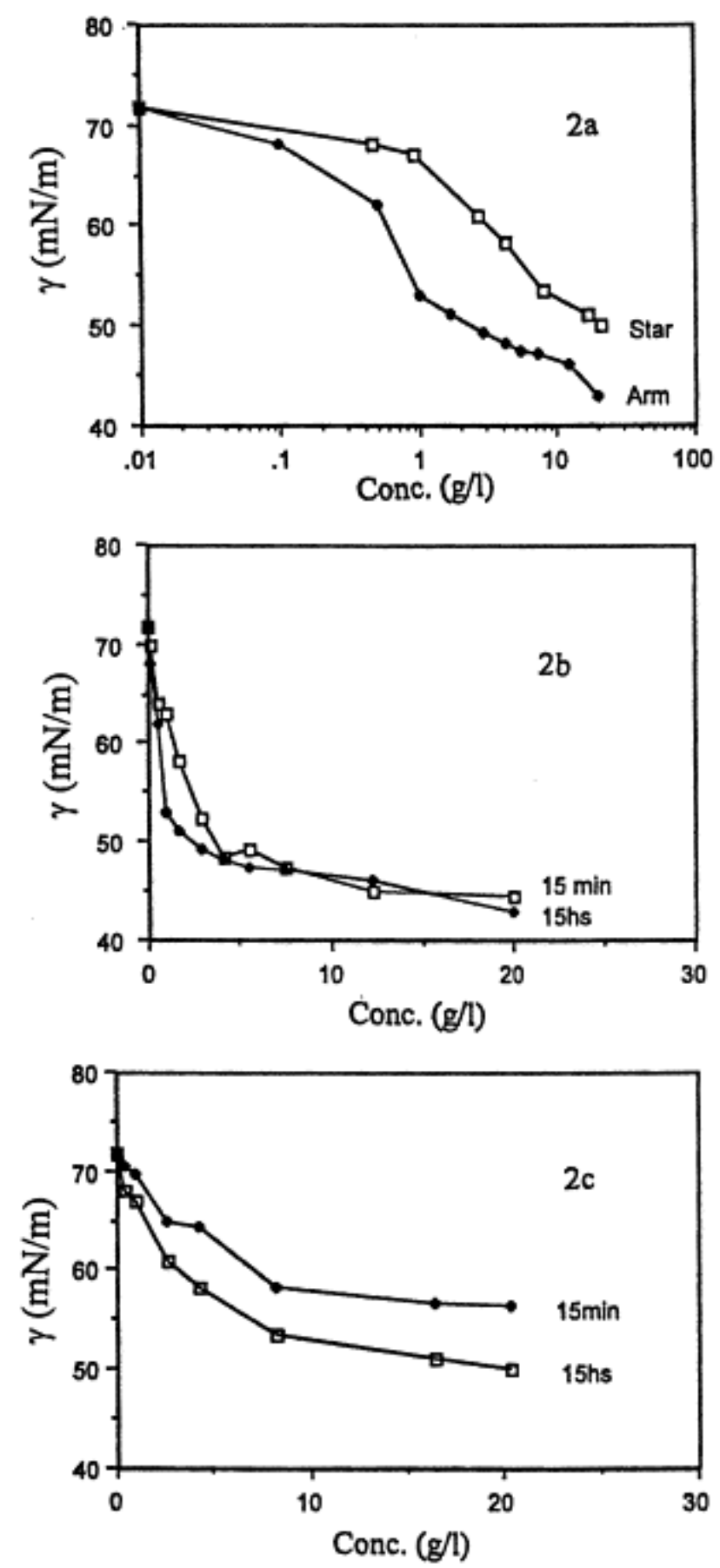

Fig. 2. Dependence of the surface tension on the copolymer concentration $\left(\mathrm{AQ}_{2}\right.$ and $\left.\mathrm{SQ}_{2}\right)$ : (a) measurements after $15 \mathrm{~h}$ for $\mathrm{AQ}_{2}$ and $\mathrm{SQ}_{2}$; (b) measurements after 15 min and $15 \mathrm{~h}$ for $\mathrm{AQ}_{2}$; (c) measurements after 15 min and $15 \mathrm{~h}$ for $\mathrm{SQ}_{2}$.

The effect of the equilibration time on the concentration dependence of the surface tension has been studied. No effect is, however, observed in case of $\mathrm{AQ}_{2}$ (Fig. 2b). In sharp contrast, the surface tension of the $\mathrm{SQ}_{2}$ solution depends on the equilibration time (Fig. 2c), the surface tension being clearly shifted to lower values at longer equilibration times. Indeed, the surface tension observed at high concentrations $(20 \mathrm{~g} / \mathrm{l})$ is decreased from 58 to $50 \mathrm{mN} / \mathrm{m}$ when the equilibration time is increased from $15 \mathrm{~min}$ to $15 \mathrm{~h}$. This effect can be rationalized by the very difficult reorganization of the $\mathrm{SQ}_{2}$ molecules in order to expose the non-ionic component at the air/water 
interface. This reorganization is hindered by the molecular structure of $\mathrm{SQ}_{2}$, in which PMMA forms a permanent core surrounded by mutually repelling charged PQDMAEMA blocks.

DLS data clearly support the structure of prefabricated micelles for $\mathrm{SQ}_{2}$ (Fig. 3). Indeed, the CONTIN size distribution at low $\mathrm{SQ}_{2}$ concentration shows micelle-like objects with an average hydrodynamic diameter of $4 \mathrm{~nm}$ (Fig. 3a). At higher concentration, these micelles are aggregated into larger structures ( Fig. 3b). In contrast, no self-assembly of the $\mathrm{AQ}_{2}$ arm is observed by DLS at concentrations lower than $0.15 \mathrm{~g} / 1$, thus in agreement with the cmc reported by surface tension measurements at $\mathrm{ca} 0.16 \mathrm{~g} / \mathrm{l}$. The good agreement in cmc measured by surface tension and DLS for the $\mathrm{AQ}_{2}$ sample must be pointed out.
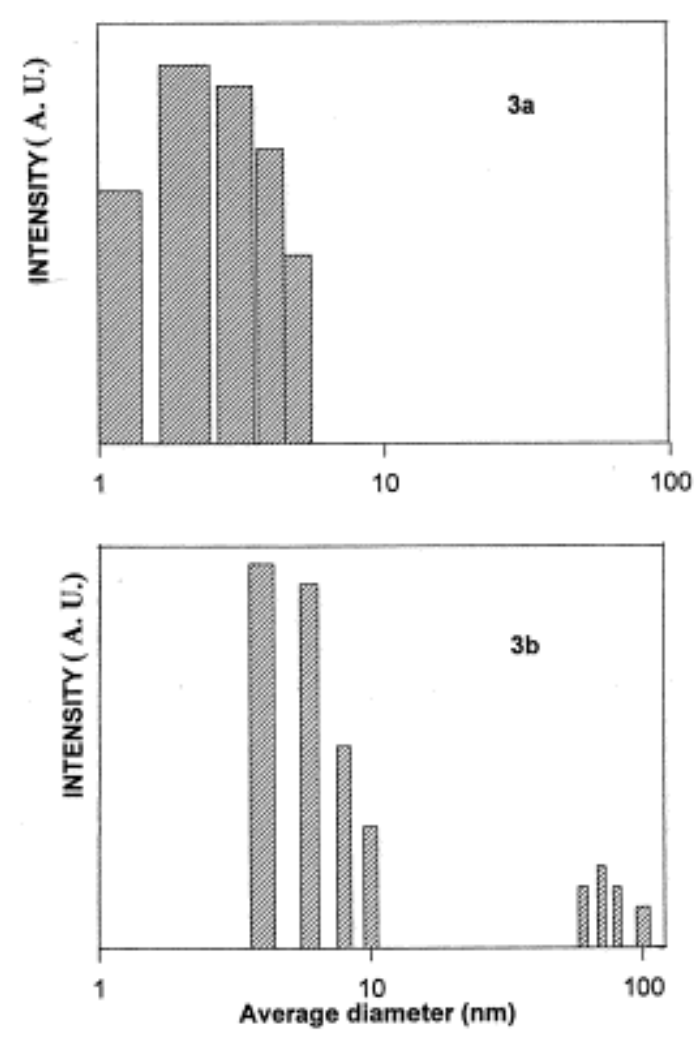

Fig. 3. CONTIN size distribution observed for the SQ2 copolymer at $25^{\circ} \mathrm{C}$ : (a) $0.5 \mathrm{~g} / \mathrm{l}$ and (b) $7 \mathrm{~g} / \mathrm{l}$.

\section{Conclusions}

Low molecular weight PQDMAEMA- $b$-PMMA copolymers containing a very small PMMA block have been dissolved in water to form micelles as close to the thermodynamic equilibrium as possible. These copolymers are characterized by a well-defined, time-independent critical micellar concentration. The size of the micelles is considerably larger for the PQDMAEMA- $b$-PMMA diblock compared to the non-quaternized counterpart as a result of the strong electrostatic repulsion of the ammonium groups, which causes the stretching of the PQDMAEMA chains in the corona. These electrostatic interactions must be partially screened by a salt in order to observe micellization. Micelles are also observed in case of a higher molecular weight, diblock although no clear cmc is detected. The effect of the length of the quaternizing alkyl bromide has also been examined. For long alkyl chains, an additional aggregation process takes place, and a behavior reminiscent to polysoap is observed. Star-shaped diblocks with PMMA forming the core are nothing but prefabricated micelles, which show low surface activity and no detectable cmc. A substantial decrease in the surface tension is, however, observed after long equilibration time, consistent with the idea that these prefabricated micelles need time to reorganize at the air/water interface. 


\section{Acknowledgements}

The authors are very much indebted to the 'Services Fédéraux des Affaires Scientifiques, Techniques et Culturelles' for financial support in the frame of the 'Pôles d'attraction Interuniversitaires: 4-11: Chimie Supramoléculaire et Catalyse Supramoléculaire'.

\section{References}

[1] Tuzar Z, Krachtovil P. In: Matijevic E, editor. Surface and colloid science. New York: Plenum Press, 1993.

[2] Chu B. Langmuir 1995;11:414.

[3] Alexandridis P. Curr Opin Colloid Interface Sci 1996; 1:490.

[4] Moffit M, Khougaz K, Eisenberg A. Ace Chem Res 1996;29:95.

[5] Winzor CL, Mrazek Z, Winnik MA, Croucher RD, Riess D. Eur Polym J 1994;30:121.

[6] Creutz S, Jerome R, Kaptijn GMP, van der Wert AW, Akkerman JM. J Coating Technol 1998;70(883):41.

[7] Yokoyama M, Inoue S, Kataoka K, Yui N, Sakurai Y. Makromol Chem Rapid Commun 1987;8:431.

[8] Yokoyama M, Okano T, Sakurai Y, Ekimoto H, Shibazaki C, Kataoka K. Cancer Res 1991;51:3229.

[9] Bedells AD, Arafeh RM, Yang Z, Attwood D, Collet JH, Price C, Booth C. J Chem Soc Faraday Trans 1993;89:1235.

[10] Luo YZ, Nicholas CV, Attwood D, Collet JH, Price C, Booth C, Chu B, Zhou ZK. J Chem Soc Faraday Trans 1993;89:539.

[11] Nicholas CV, Luo YZ, Deng NJ, Attwood D, Collet JH, Price C, Booth C. Polymer 1993;34:138.

[12] Alexandridis P, Athanassiou V, Fukuda S, Hatton TA. Langmuir 1994; 10:2604.

[13] Yang L, Bedells AD, Attwood D, Booth C. J Chem Soc Faraday Trans 1992;88:1447.

[14] Deng Y, Ding J, Stubbersfield RB, Heatley F, Attwood D, Price C, Booth C. Polymer 1992;33:1963.

[15] Brown W, Schillen K, Almgren M, Hvidt S, Bahadur P. J Phys Chem 1991;95:1850.

[16] Malmsten M, Lindman B. Macromolecules 1992;25:5440.

[17] Almgren M, Bahadur P, Jansson M, Li P, Brown W, Bahadur A. J Colloid Interface Sci 1992;151:157.

[18] Linse P. Macromolecules 1993;26:4437.

[19] Mortensen K, brown W. Macromolecules 1993;26:4128.

[20] Almgren M, Brown W, Hvidt S. Colloid Polym Sci 1995;273:2.

[21] Wilhem M, Zhao CL, Wang Y, Xu R, Winnik MA, Mura JL, Riess G, Croucher MD. Macromolecules 1991;24:1033.

[22] Hruska Z, Piton M, Yekta A, Duhamel J, Winnik MA, Riess G, Croucher MD. Macromolecules $1993 ; 26: 1825$.

[23] Selb J, Gallot Y. Makromol Chem 1980;181:809.

[24] Selb J, Gallot Y. Makromol Chem 1980;181:2605.

[25] Selb J, Gallot Y. Makromol Chem 1981;182:1491.

[26] Selb J, Gallot Y. Makromol Chem 1981;182:2523.

[27] Biggs S, Vincent B. Colloid Polym Sci 1992;270:563.

[28] Gao ZS, Varshney SK, Wong S, Eisenberg A. Macromolecules 1994;27:7923.

[29] Tuzar Z, Webber SE, Ramireddy C, Munk P. Polym Prepr 1991;32:525.

[30] Cao T, Munk P, Ramireddy C, Tuzar Z, Webber SE. Macromolecules 1991;24:6300.

[31] Kiserow D, Prochazka K, Ramireddy C, Tuzar Z, Munk P, Webber SE. Macromolecules 1992;25:461.

[32] Prochazka K, Kiserow D, Ramireddy C, Webber SE, Munk P, Tuzar Z. Makromol Chem Macromol Symp 1992;58:201.

[33] Chan J, Fox S, Kiserow D, Ramireddy C, Munk P, Webber SE. Macromolecules 1993;26:7016.

[34] Tian M, Qin A, Ramireddy C, Webber SE, Munk P, Tuzar Z, Prochakza K. Langmuir 1993;9:1741.

[35] Tuzar Z, Kratochvil P, Prochakza K, Munk P. Collect Czech Chem Commun 1993;58:2362.

[36] Selb J, Gallot Y. In: Goodman I, editor. Developments in block copo- lymers, vol. 2. Amsterdam: Elsevier, 1985. p. 85 .

[37] Zhang L, Eisenberg A. Science 1995;268:1728.

[38] Zhang L, Eisenberg A. J Am Chem Soc 1996;118:3168.

[39] Zhang L, Yu K, Eisenberg A. Science 1996;272:1777.

[40] Zhang L, Shen H, Eisenberg A. Macromolecules 1997;30:1001.

[41] Zhang L, Eisenberg A. Macromolecules 1996;29:8805.

[42] Yu K, Eisenberg A. Macromolecules 1996;29:6359.

[43] Yu K, Eisenberg A. Macromolecules 1998;31:3509.

[44] Yu Y, Zhang L, Eisenberg A. Macromolecules 1998;31:1144.

[45] Yu Y, Zhang L, Eisenberg A. Langmuir 1997;13:2578.

[46] Shen H, Zhang L, Eisenberg A. J Phys Chem 1997;101:4697. 
[47] Yu K, Zhang L, Eisenberg A. Langmuir 1996;12:5980.

[48] Yu Y, Eisenberg A. J Am Chem Soc 1997;119:8383.

[49] Yu Y, Eisenberg A. Macromolecules 1998;31:5546.

[50] Baines FL, Armes SP, Billingham NC, Tuzar Z. Macromolecules 1996;29:8151.

[51] Anton P, Koberle P, Laschewsky A. Makromol Chem 1993;194:1.

[52] Binana-Limbele W, Zana R. Macromolecules 1990;23:2731.

[53] Chu DY, Thomas JK. Macromolecules 1987;20:2133.

[54] Chu DY, Thomas JK. J Phys Chem 1985;89:4065.

[55] Binana-Limbele W, Zana R. Macromolecules 1987;20:1331.

[56] Ruokolainem J, Makinen R, Torkelli M, Makala T, Serimaa R, ten Brinke G, Ikkala O. Science 1998;280:557.

[57] Antoun S, Teyssie Ph, Jerome R. Macromolecules 1997;30:1556.

[58] Zilliox JG, Rempp P, Parrod J. J Polym Sci, Part C 1968;22:145.

[59] Jae-Min O, Hyung-Jong L, Hong-Ku S, Sam-Kwon C. Polym Bull 1994;32:149.

[60] Stepanek P. In: Brown W, editor. Dynamic light scattering. London: Oxford University Press, 1972.

[61] Baines FL, Billingham NC, Armes SP. Macromolecules 1996;29:3416.

[62] Rosen MJ. Surfactant and interfacial phenomena. New York: Wiley, 1989.

[63] Piirma I. Br Polym J 1989;21:1.

[64] Strauss UP, Jackson EG. J Polym Sci 1951;6:649.

[65] Macowsko CW, Guegan P, Khandpur AK, Nakayama A, Marechal P, Inoue T. Macromolecules 1996;29:5590.

[66] Khougaz K, Astafieva I, Eisenberg A. Macromolecules 1995;28: 7135.

[67] Zhang L, Barlow RJ, Eisenberg A. Macromolecules 1995;28:6055.

[68] Pincus P. Macromolecules 1991;24:2912.

[69] Huynh-Ba G, Jerome R, Teyssie Ph. Colloid Polym Sci 1979;257:1294.

[70] Huynh-Ba G, Jerome R, Teyssie Ph. J Polym Sci, Polym Phys Ed 1980;18:2391.

[71] Nitsch W, Kremnitz W, Schweyer G, Bunsen-Ges B. Phys Chem 1978;91:218.

[72] Powney J. Trans Faraday Soc 1935:1510.

[73] Adam NK, Shute HL. Trans Faraday Soc 1938:758. 